\title{
WAVE PROPAGATION AT THE INTERFACE OF A TWO-LAYER FLUID SYSTEM IN THE LABORATORY
}

\author{
Chen-Yuan Chen \\ Department of Management Information System, Yung-Ta Institute of Technology and Commerce, Ping-Tung County, \\ Taiwan 90942, R.O.C. \\ John Rong-Chung Hsu \\ Department of Marine Environment and Engineering, National Sun YatSen University, Kaohsiung, Taiwan 80424, R.O.C. \\ NCKU-NSYSU Research Center for Ocean Environment and Technology, Taiwan; Honorary Research Fellow, School of \\ Civil and Resource Engineering, University of Western Australia, WA 6010, Australia.

\section{Cheng-Wu Chen \\ Department of Logistics Management, Shu-Te University, Kaohsiung County, Taiwan 82445, R.O.C., cwchen@mail.stu.edu.tw} \\ Ching-Feng Kuo \\ Department of Color Filter Inspection, Chi Mei Optoelectronics, Tainan County, Taiwan, R.O.C. \\ Hsin-Hsun Chen \\ Graduation from Institute of Physical Oceanography, National Sun Yat-Sen University, Kaohsiung, Taiwan 80424, \\ R.O.C.
}

See next page for additional authors

Follow this and additional works at: https://jmstt.ntou.edu.tw/journal

Part of the Oceanography Commons

\author{
Recommended Citation \\ Chen, Chen-Yuan; Hsu, John Rong-Chung; Chen, Cheng-Wu; Kuo, Ching-Feng; Chen, Hsin-Hsun; and Cheng, Min-Hung \\ (2007) "WAVE PROPAGATION AT THE INTERFACE OF A TWO-LAYER FLUID SYSTEM IN THE LABORATORY," Journal of \\ Marine Science and Technology. Vol. 15: Iss. 1, Article 2. \\ DOI: 10.51400/2709-6998.2027 \\ Available at: https://jmstt.ntou.edu.tw/journal/vol15/iss1/2 \\ This Research Article is brought to you for free and open access by Journal of Marine Science and Technology. It has been \\ accepted for inclusion in Journal of Marine Science and Technology by an authorized editor of Journal of Marine Science and \\ Technology.
}




\section{WAVE PROPAGATION AT THE INTERFACE OF A TWO-LAYER FLUID SYSTEM IN THE LABORATORY}

\section{Acknowledgements}

The authors are grateful for the research grants to JRCH from the National Science Council, Republic of China under Grant No. NSC 90-2611-M-110-014 and NSC 91-2611-M-110-004. They wish to thank Dr. H.

Michallet, Laboratoire des Ecoulements Geophysiques et Industriels (LEGI), Institute National

Polytechnique de Grenoble, France for his assistance in acquisition of the ultrasonic probes used in this study as well as MNDAS software system supplied by Tainan Hydraulics Laboratory, National Cheng Kung University, Taiwan.

\section{Authors}

Chen-Yuan Chen, John Rong-Chung Hsu, Cheng-Wu Chen, Ching-Feng Kuo, Hsin-Hsun Chen, and MinHung Cheng 


\title{
WAVE PROPAGATION AT THE INTERFACE OF A TWO-LAYER FLUID SYSTEM IN THE LABORATORY
}

\author{
Chen-Yuan Chen*, John Rong-Chung Hsu**,***, Cheng-Wu Chen****, \\ Ching-Feng Kuo ${ }^{* * * * *}$, Hsin-Hsun Chen******, and Min-Hung Cheng**
}

Key words: ultrasonic probe, wavelet, denoise, calibrations, data acquisition.

\section{ABSTRACT}

A series of laboratory experiments on internal solitary wave (ISW) had been conducted in a two-layer fluid system. Internal solitary waves were generated in laboratory which was controlled by several variables, including different layer thickness ratios $H_{1} / H_{2}$, interface differences $\eta_{o}$, and the density ratios $\rho_{1} / \rho_{2}$. The wave flume used in this experiment was 12 meter long with a cross-section $0.5 \mathrm{~m}$ wide by $0.7 \mathrm{~m}$ in height. Five ultrasonic probes placed at equidistance recorded the interface fluctuations, and one density probe measured the change of density across the interface. Data collection and processing were carried out via control software. This paper presents the results of the physical properties related to propagation and dissipation of the ISW. It appears that the leading soliton feature occurred while layer thickness ratios $\left|H_{1} / H_{2}\right|$ was large and the decaying ratio in energy and wave amplitude could be approximated by a linear regression trend with distance.

\section{INTRODUCTION}

Internal gravity waves (IGW) are wave motion in the interior of the ocean. They occur in a water body consisting of layers of different densities, mostly due to difference in water temperature or salinity. In most

Paper Submitted 07/07/05, Accepted 09/13/05. Author for Correspondence: Cheng-Wu Chen.E-mail: cwchen@mail.stu.edu.tw.

*Department of Management Information System, Yung-Ta Institute of Technology and Commerce, Ping-Tung County, Taiwan 90941, R.O.C.

**Department of Marine Environment and Engineering, National Sun Yat-

Sen University, Kaohsiung, Taiwan 80424, R.O.C.

***NCKU-NSYSU Research Centerfor Ocean Environment and Technology, Taiwan; Honorary Research Fellow, School of Civil and Resource Engineering, University of Western Australia, WA 6009, Australia.

****Department of Logistics Management, Shu-Te University, Kaohsiung County, Taiwan 82445, R.O.C.

*****Department of Color Filter Inspection, Chi Mei Optoelectronics, Tainan County, Taiwan, R.O.C.

******Graduation from Institute of Physical Oceanography, National Sun Yat-Sen University, Kaohsiung, Taiwan 80424, R.O.C. cases, the vertical structure of water density in the ocean can be approximated by a two-layer system. Similar to the surface gravity waves, their counterpart fluctuating on the ocean surface that is at the interface of water and air, IGWs occur at the interface between two fluid layers of different densities. As early as in 1970s Cacchione and Southard [4] discussed the potential significance of shoaling IGWs on causing sediment movement along continental shelves and slopes. Among the prevailing wave acting in the ocean, ISWs have been claimed to affect oil-drilling operations and increase scour and sediment transport on the seabed in deep water. Recent studies have also supported the potential capability of the ISW in resuspending and transporting sediment $[2,3,14,16]$.

However, it is difficult, though not impossible, to thoroughly investigate the characteristics of internal waves in field observations. Therefore, alternative approach using laboratory facilities to study IGW have become $^{1}$ [11]. For example, Segur and Hammack [15] and Kao et al. [10] have created IGWs in a continuously stratified fluid composed of brine and water. They also characterized ISW in terms of the shape of wave profile and the amplitude-wavelength relationship. Validation of ISW in small amplitudes [10-12,15] and large amplitude [13] have been reported. Chen et al. [5-8] generated internal waves in a two-layer stratified fluid of fresh and brine water. They characterized the generation of an ISW. Furthermore, this paper extends the investigations on ISW propagation in constant water depth in a wave tank. Data collection and processing were completed by MNDAS (Multi Nodes Data Acquisition System). In addition, wave dissipation due to bottom friction and viscous effect is presented in this paper.

\footnotetext{
${ }^{1}$ a viable means on internal solitary waves.
} 


\section{EXPERIMENTAL SETUP}

Experimental procedure to produce an ISW in a two-layered fluid system is reported in an accompanying paper by Chen et al. [5]. The apparatus for measuring wave propagation in the interface is in this paper, as well as the physical proportion.

\section{Ultrasonic probes}

Ultrasonic probes were used to record internal wave fluctuations, while capacitance gauges for surface wave variations. The analog signals of these waves were processed by MNDAS data acquisition system to produce digital outputs of time series in wave height. Each ultrasonic probe consisted of an electronic fibreglass printed circuit board fabrication on a fixed piezoelectric ceramic. Two piezoelectric ceramics were approximately $15 \mathrm{~cm}$ apart, with one of them to emit ultrasound signals and the other to receive and reflect the pulse. The basic configuration of ultrasonic probe linking the interface displacement is shown schematically in Figure 1. The principle of operation was based on the fact that the speed of acoustic wave was constant in a homogeneous fluid and its variations depended on the density and elastic properties of the medium if passed. The speed increased with fluid density; consequently the phase shift between an emitted and reflected acoustic wave was related to the travelling time if exhibited to the interfacial position. As the speed of an acoustic wave increased with fluid density, the relative location of the interface between the ceramics was determined by the travelling time of the ultrasonic wave packet. The interface was required to be at least $2 \mathrm{~cm}$ from the sensors in order for the response to remain linear. If the interface was closer to the emitting ceramic at the top, the travelling time of the ultrasonic wave packet would be longer than that closer to the reflecting ceramic. If the travelling time for the (a)

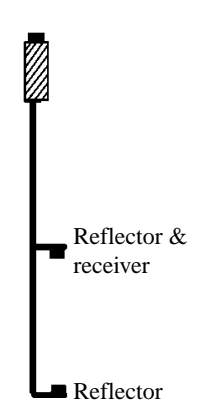

(b)

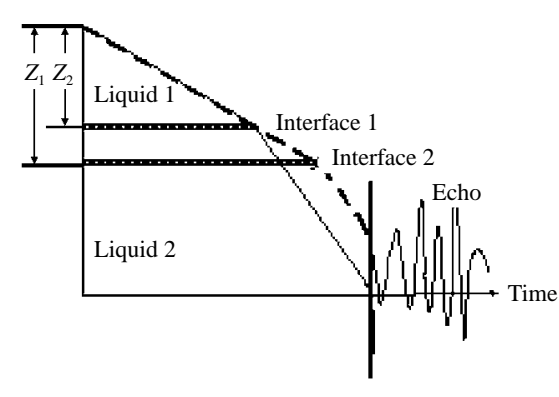

Fig. 1. (a) Basic construction of an ultrasonic probe; (b) Diagram of sound propagation in a two-layer fluid system. sound packets was very small in comparison with the time scale of the interface displacement, the interfacial position was considered us constant over this time interval. If the sonic packets were constantly transmitted across the interface, then a constantly changing travel time resulted if the interface was oscillating. Each travel time was then converted into a voltage by an electronic circuit corresponded to the position of the interface. Consequently, through calibrating a probe, it was possible to calculate the interface elevation with time.

After the thickness of the interface diffusion became large due to mixing after two or more test runs, the ultrasonic probe still measured the boundary of the fluids resulting in apparent interface oscillations. Each ultrasonic probe was supported by a carriage holder on rails at the top of the wave flume (seen in Figure 2). Six pairs of ultrasonic probes and capacitance wave gauges were connected to an amplifier unit and A/D converter, finally the digital signals of the internal waves were processed by "MNDAS" software resided in a personal computer. Probes 1-5 were equidistantly placed in the constant water depth section in the flume on the lefthand-side of the movable vertical gate, to measure the interface displacement as a wave propagated in the flume and to calculate wave speed. Each gauge was mounted on a sliding scale and calibration was carried out by lowering and raising the probe and recording voltage output on each movement.

\section{Ultrasonic density probe}

The structure of a density probe is similar to ultrasonic probe, comprising emitter and receiver points at an identical plane surface. An ultrasonic density probe for liquids is placed at the interface of a two-layer fluid. From the acquired measurements of sound speed and acoustic impedance, the physical properties of density profile could be calculated whilst internal waves propagate. The density of the stratified fluid system was in the range of 999 to $1030 \mathrm{~kg} / \mathrm{m}^{3}$ at normal room temperature.

\section{Data acquisition system}

Multi Nodes Data Acquisition System (MNDAS) was a high performance data acquisition system which was a process control software developed by Tainan Hydraulics Laboratory, National Chen-Kung University in Taiwan. This system allowed simultaneous data collection from multiple channel nodes, including ultrasonic probes, capacitance wave gauges, density probe, and velocimetey probe. The MNDAS included a variety of sources and receivers components 
for completing a series of tasks of acquiring, processing, analyzing, and displaying dynamic signals. In comparison with other popular software (see Table 1), such as LabVIEW and LabTech Notebook, MNDAS, being designed using low-level programming, was much easier to operate. By means of an analog-to-digital (A/D) converter, data were downloaded to Windows platform from raw media format in binary file to data format in ASCII file.

\section{Oscilloscope}

Pre-inspection of an ultrasonic signal was performed using the standard procedure of "Normal beam

Table 1. Comparison among software for data acquisition

\begin{tabular}{|c|c|c|}
\hline Software type & Advantages & Disadvantages \\
\hline $\begin{array}{l}\text { LabVIEW } \\
\text { (Laboratory virtual } \\
\text { instrument engineering } \\
\text { workbench) }\end{array}$ & $\begin{array}{l}\text {-Icon-driven interface } \\
\text { •Powerful graphical editing facilities } \\
\text {-Selecting appropriate functional blocks }\end{array}$ & $\begin{array}{l}\text {-Additional effort required to design a program to } \\
\text { record and graph the digital input signal in real-time } \\
\text {-Expensive (about USD } \$ 2000 \text { ); specialized converted } \\
\text { card for operation }\end{array}$ \\
\hline $\begin{array}{l}\text { Lab tech notebook } \\
\text { (Laboratory } \\
\text { technologies notebook) }\end{array}$ & $\begin{array}{l}\text {-Icon-driven interface; no programming } \\
\text { required } \\
\text {-Simultaneous support for multiple boards } \\
\text { - Real time data acquisition, data display, } \\
\text { multitasking and datalogging }\end{array}$ & $\begin{array}{l}\text {-Expensive (about USD } \$ 1000 \text { ) } \\
\text { - Technical training time- consuming }\end{array}$ \\
\hline $\begin{array}{l}\text { MNDAS } \\
\text { (Multi nodes data } \\
\text { acquisition system) }\end{array}$ & $\begin{array}{l}\text { - Collection of analog data from multi- } \\
\text { channel } \\
\text { - Reduce complicated circuit arrangement } \\
\text {-Easy to learn }\end{array}$ & $\begin{array}{l}\text {-No data display in real time } \\
\text { - Only display four panels simultaneously }\end{array}$ \\
\hline
\end{tabular}

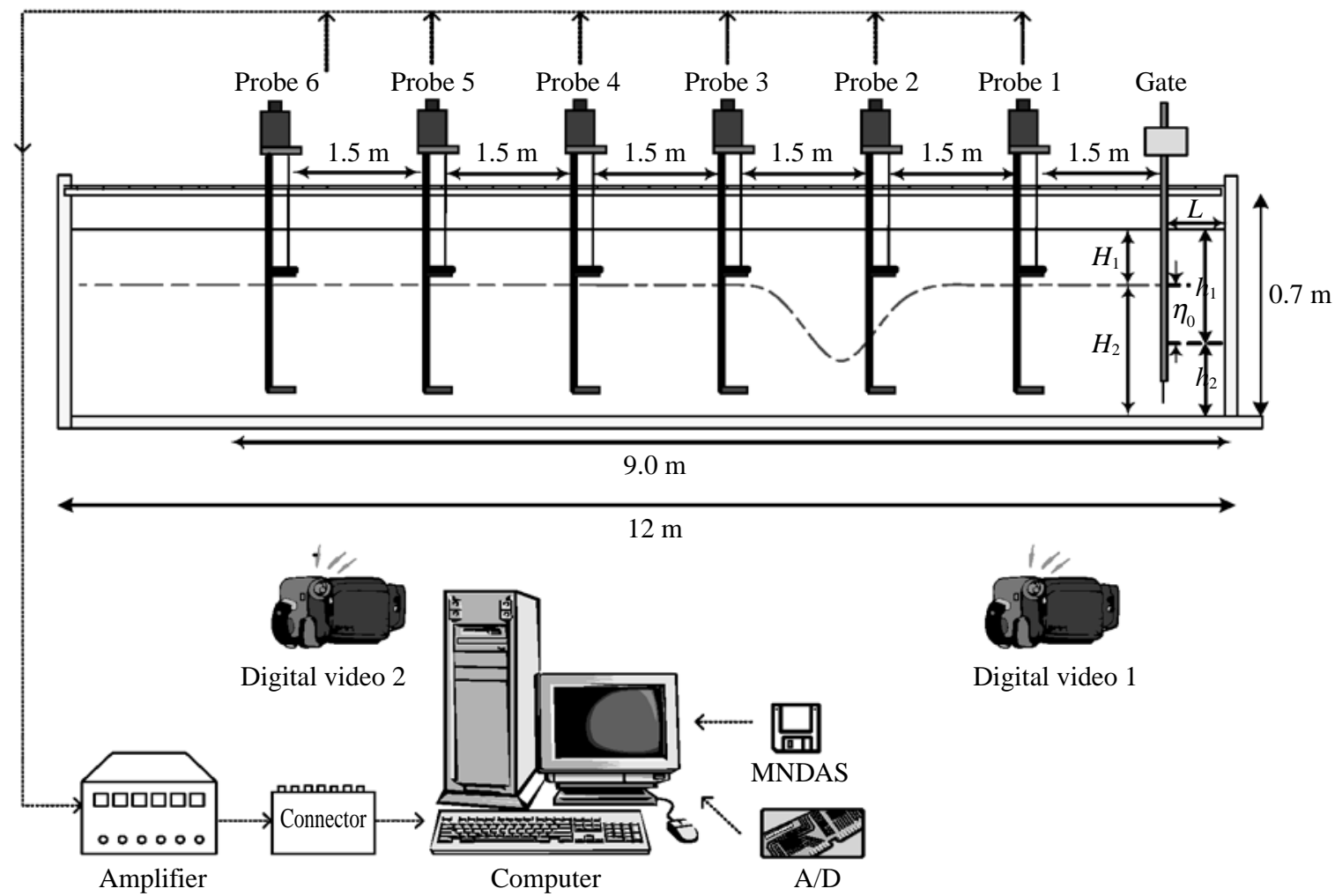

Fig. 2. A schematic diagram showing the experimental set-up for the present study. 
Pulse - Echo" measurement. This integrated data acquisition and waveform analysis system necessarily consisted of ultrasonic instrument, a digital storage oscilloscope and a software package for analyzing the acquired ultrasonic waveform signal. A digital storage oscilloscope processed target echoes with their amplitude and location using a built in "TDS202", developed by Tektronix Corporation. This allowed signals from 4 channels in $200 \mathrm{MHz}$ bandwidths and sampling rates up to $2 \mathrm{GS} / \mathrm{s}$, be utilized to calibrate and adjust the receiver waveform.

\section{DATA ANALYSIS}

Time series data were obtained for measurement of a particular variable from capacitance, density and ultrasonic probes. The data analysis of internal waves included data collection, wave filter, calibrations and processing.

\section{Collection}

The analog voltage output recorded by each capacitance or ultrasonic probe was sampled at constant time intervals. By means of cables, output voltage signals were sent directly to a personal computer. These analog voltages were transmitted to an analog-to-digital port, and stored in the computer files with text format using the MNDAS software. These were original files containing interface displacements including surface, internal and density profiles. Prior to each test run, probes were calibrated to allow the voltages measured by them to be converted into actual units. Calibration before each run also ensured the data obtained was as accurate as possible. Data was acquired at the sampling rate of $25 \mathrm{~Hz}$ for all tests; however, duration of each particular run varied with the characteristics, such as phase speed or wave amplitude. Generally speaking, internal wave activity normally lasted a long period, often exceeding five minutes.

\section{Denoise}

All possible filtering combinations (low- and highpass) were performed to decompose a time series derived from data collection described above. Wavelet Toolbox in MATLAB was adopted, taking advantage of its efficiency to filter high frequency signal produced by electronic instruments (e.g. circuits, channels, grounding lines, and A/D converter etc). By applying functions of decomposition, denoise and reconstruction of wavelet theory, abnormal signals could be eliminated and the appropriate rating curve of water level thus modified. With multiresolution analysis (MRA) and

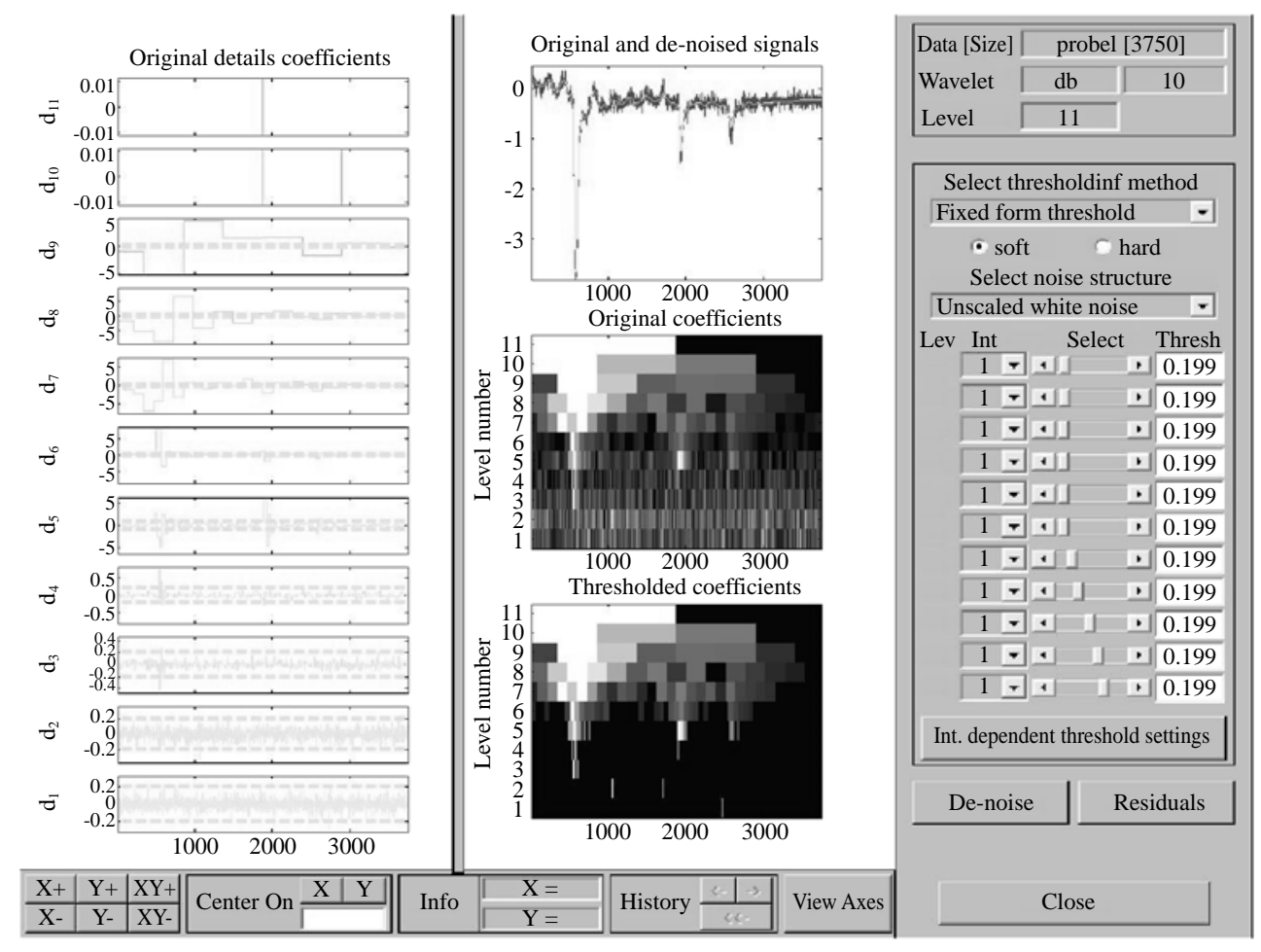

Fig. 3. A screen frame revealed the functions in a MATLAB wavemenu. 
wavelet function Daubechies (db), high frequency content was removed and a new "de-noise" signal reconstructed using inverse wavelet transform. Figure 3 shows a menu in MATLAB for accessing the various graphical tools provided in the Wavelet Toolbox graphical user interface (GUI) tools. A frame reveals the function on one-dimension discrete wavelet analysis tools in a MATLAB wavemenu. The middle top panel shows the contrast between the original and de-noise signals with different coloring line. On the left-handside panel, interior dependent threshold settings can be arbitrarily modulated by wavelet function; on the righthand-side panel, manual threshold settings are supported.

\section{Calibrations}

Calibration was used to determine the correlation between an input and an output quantity. All measurement instruments (including density probes, ultrasonic probes, and capacitance gauges, etc.) had to be calibrated. Herein, outputs from probes which measured the fluctuation at the surface, interface, density elevation were in volts. Calibration for these probes was essential in order to convert voltages into interface displacement as seen in Figure 4. The ultrasonic probes were calibrated by lowering and raising them relative to the interface within $5 \mathrm{~cm}$ intervals and recording the voltage change respectively. This enabled a calibration curve to be constructed and gave the gain in voltage for a given interface displacement, according to the relationship of a linear regression (Figure 4). The relation can be represented by $\Delta h=\Delta V_{s} / \alpha$, where $\alpha$ is an experimentally determined coefficient, $\Delta V_{s}$ is the change in voltage and $\Delta h$ is the corresponding change

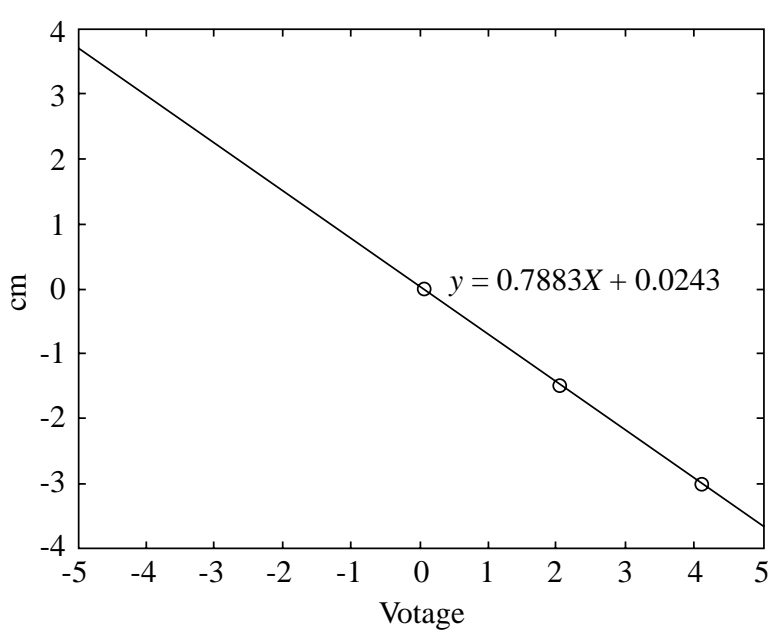

Fig. 4. A calibration curve showing interface displacement against digital voltage output with a linear regression fit. in the interface level. The output voltage from a probe was no more than 5 volts for small fluctuation, and sometimes exceeded it for large fluctuation. The location of the interface was indispensable to be at least $2 \mathrm{~cm}$ from the sensors in order for the response to remain linear.

\section{Processing}

Each time series data was defined as a succession of observations made through time, where time was the independent variable and the value observed is the dependent variable (Hegge and Masselink, 1996). Individual data record matriculated by the capacitance and ultrasonic probes was therefore a time series, where the dependent variable was the surface and internal elevations. As a result of the data analysis mentioned above, data files from each test run were transformed into interface elevation plots (Figure 5). Recorded internal wave forms in consecutive runs in which the experiment control condition were $H_{1} / H_{2}=10 \mathrm{~cm} / 30$ $\mathrm{cm}, \eta_{o}=h_{1}-H_{1}=5 \mathrm{~cm}$. Each waveform was processed by a series of (a) analog/digital converted voltages, (b) wavelet filter, (c) calibration, and (d) de-mean. Repeatability of the wave generation was necessary to ensure reliable wave production. By means of the plot in Figure 5 (d), wave profile can be utilized to evaluate the wave characteristics using MATLAB programs.

\section{EXPERIMENTAL RESULTS ON WAVE PROPAGATION}

After a sufficient distance from its source, an internal wave reached a stable form and propagated in
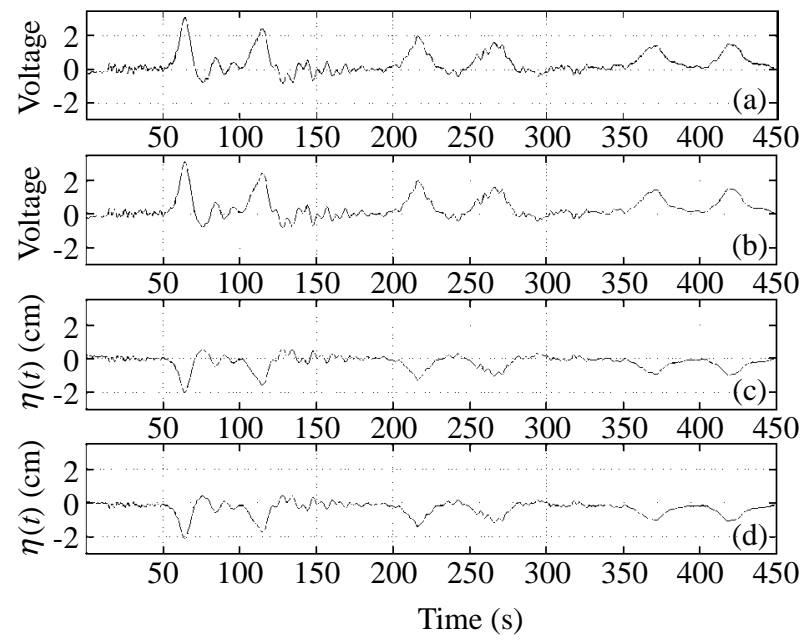

Fig. 5. Time series of (a) analog/digital converted voltages; (b) wavelet filter; (c) calibration and (d) de-mean. 
the main section of constant depth in the wave flume. The fluctuations of the wave were recorded using five ultrasonic probes placed at pre-arranged positions, shown in Figures 2 and 6. On the top of Figure 2, the wave probes were shown at exact positions. A dashed line in negative slant marks the propagation of the ISW, while a dashed line in positive slant is for reflected waves. Because the slope of a dashed line is proportional to wave speed, if represent, a variation in wave decay. Therefore, Figure 6 illustrates not only the time series of wave propagation and reflection along the flume for the five probes identified but also their corresponding speeds of propagation. The initial peak at probe $\mathrm{P} 1$ was approximately $2.2 \mathrm{~cm}$ high, reduced to about $1.7 \mathrm{~cm}$, while propagating all the way down to probe P5. Upon encountering the end of the wave flume, wave heights decreased slightly with its peak widen. However, the reflected peak remained approximately $1.1 \mathrm{~cm}$ in height after returning to probe $\mathrm{P} 1$ position. The dashed lines mark the route of a leading IGW from P1 to P5 and eventually reflected back and forth in the flume. The gradient of the slant lines indicate wave speed was about $12.3 \mathrm{~cm} / \mathrm{s}$ for the incident wave and $11.6 \mathrm{~cm} / \mathrm{s}$ for reflected wave during its first journey. Referring to the wave profile recorded at second probe P2 in Figure 6, the time intervals between a reflecting peak and successive incident peak after reflection from the RHS of the flume increased with time, compared to that recorded at $\mathrm{P} 1$, because P2 was located $3 \mathrm{~m}$ from the movable gate. The journey for the leading solitary wave to travel was $18 \mathrm{~m}$ approximately.

At the beginning and the end of each test run, the vertical distribution of fluid density was measured by a density probe at the middle section of the wave flume.

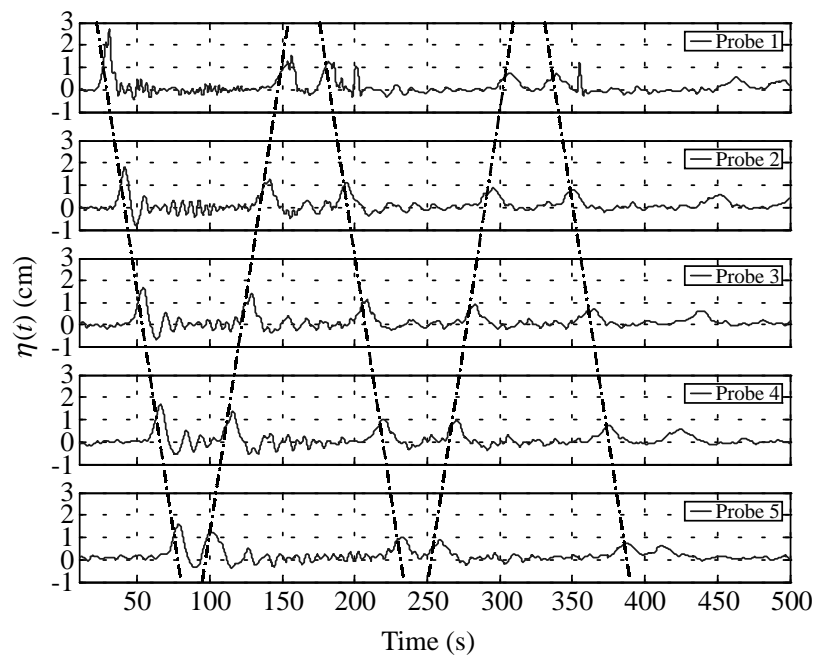

Fig. 6. Time series of IGW profile at five fix positions along a wave flume.
The vertical distribution of density profile Figure 7 showed a near perfect two-layer system of fresh-brine water before first run (symbol $\mathrm{O}$ ), and diffused state after five runs $(\Delta)$. These indicates the density variation in the vertical direction and the abscissa is the ratio of density difference between its ambient and the upper layer fluid to that between the two layers before and after each run, where the ordinate at $z=0 \mathrm{~cm}$ was the original interface (defined as positive upward). In all cases the initial thickness of the pycnocline was within or much less than $1 \mathrm{~cm}$; experiments were terminated when this thickness became about $4-5 \mathrm{~cm}$ and the fluid in the flume had to be drained completely. Diffusion of fluid at the interface due to disturbances and mixing was apparent after a test run.

\section{Waveform}

Based on the thickness ratio $H_{1} / H_{2}$ in a stratified two-layer system, ISW form can be categorized as either elevation or depression type. Examples of the measured waveform for three different depth parameters $\left|H_{2}-H_{1}\right| / H$ are presented in Figure 8. In this figure, wave profile fluctuations are nondimensionalised $\eta(t)$ by the maximum amplitude of the incident wave ai in each test run. For $H_{1} \neq H_{2}$, the peaks of the leading IGW above or below the interface baseline of $\eta(t) / a_{i}=0$ yielded a downstroke or upstroke overshooting from the baseline and generated a trough or crest that formed the beginning of the trailing oscillations. This phenomenon was obvious especially at "critical depth", a condition of approximately equal layer thickness (i.e. $H_{1}=H_{2}$ ). Otherwise, ISW of elevation type was observed if $H_{1}>H_{2}$ (i.e. $H_{1} / H_{2}=30$ /

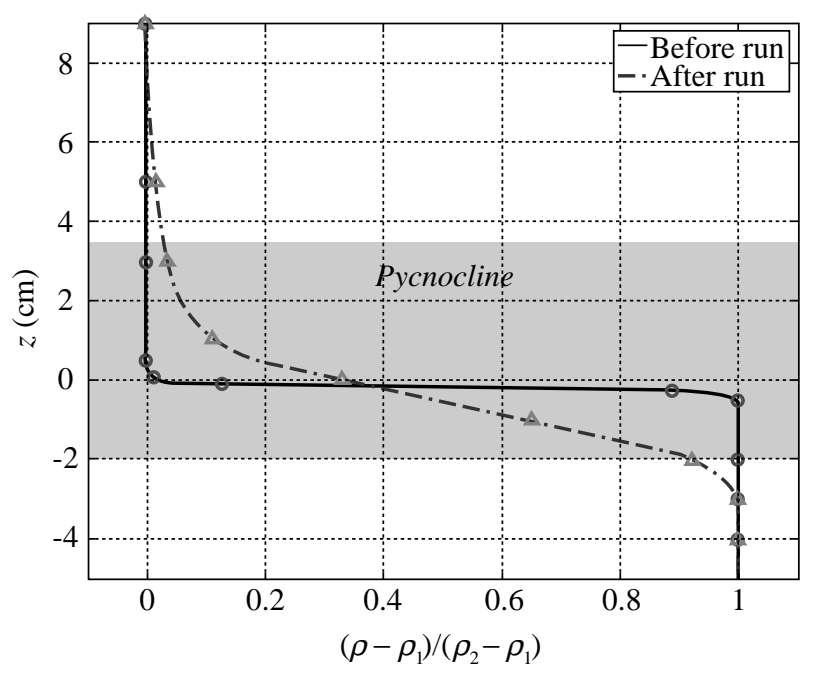

Fig. 7. Vertical distribution of density profile. 
Table 2. Decay of internal wave amplitude in a wave flume

\begin{tabular}{lcc}
\hline & Wave loss/distance $(\mathrm{m})$ & Decaying rate $\left(\mathrm{m}^{-1}\right)$ \\
\hline Koop and Butler [11] & $70 \% / 7 \mathrm{~m}$ & $84 \%$ \\
Michallet and Barthelemy [12, 13] & $50 \% / 3.56 \mathrm{~m}$ & $82 \%$ \\
Ariyaratnam [1] & $80 \% / 8 \mathrm{~m}$ & $97 \%$ \\
Present study & $86-88 \% / 6 \mathrm{~m}$ & $97.5 \%$ \\
\hline
\end{tabular}

Note: water-petroleum used in [1] and [12, 13], but fresh-brine water in ariyaratnam and present study.
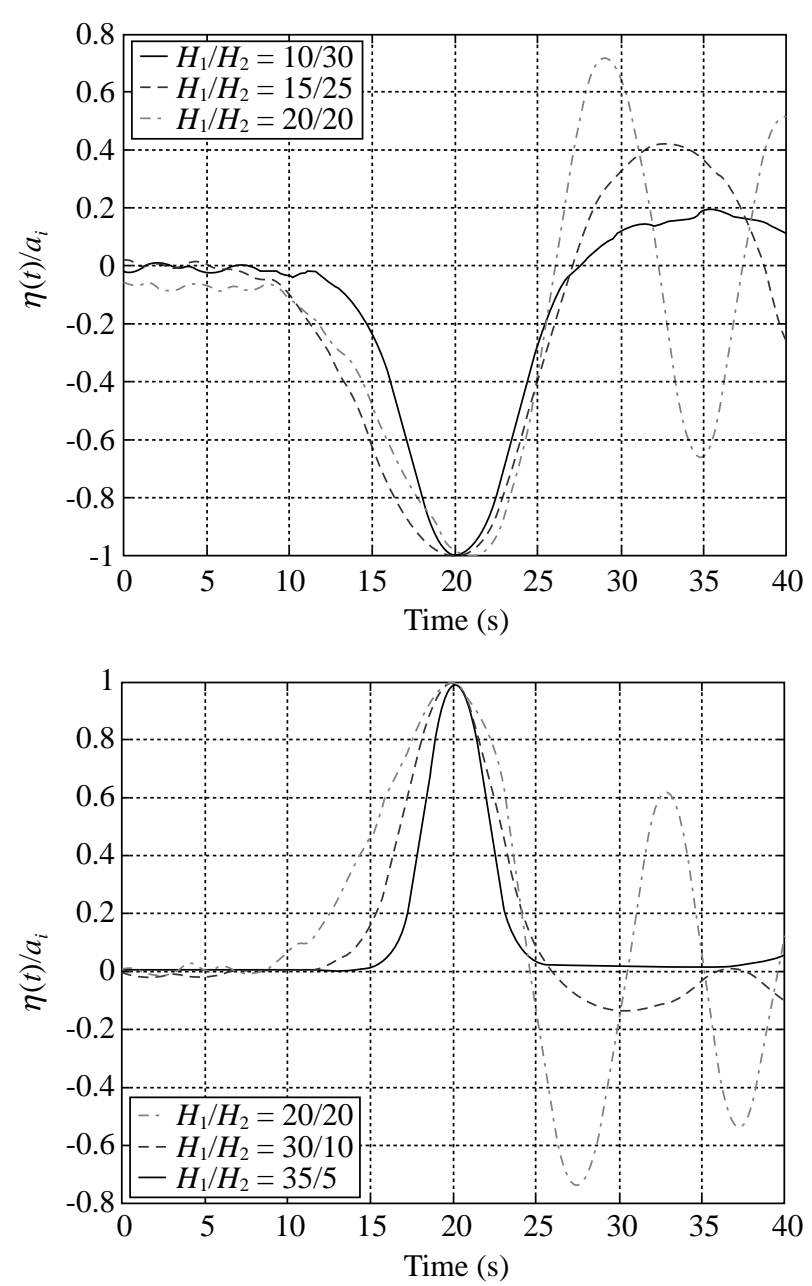

Fig. 8. Typical wave profiles for stable/unstable internal waves of depression and elevation types, showing dimensionless interface displacement versus time for three different stratification depth ratios of (a) $H_{1} / H_{2}=10 / 30,15 / 25,20 / 20$; and (b) $H_{1} / H_{2}=$ $35 / 5,30 / 10,20 / 20$.

10 or 35/5); and vise versa, of a depression type when $H_{1}$ $<H_{2}$ (i.e. $H_{1} / H_{2}=10 / 30$ or $15 / 25$ ). In the ocean environment, for example, soliton feature of an internal wave is more obvious in deep water than that in conti-

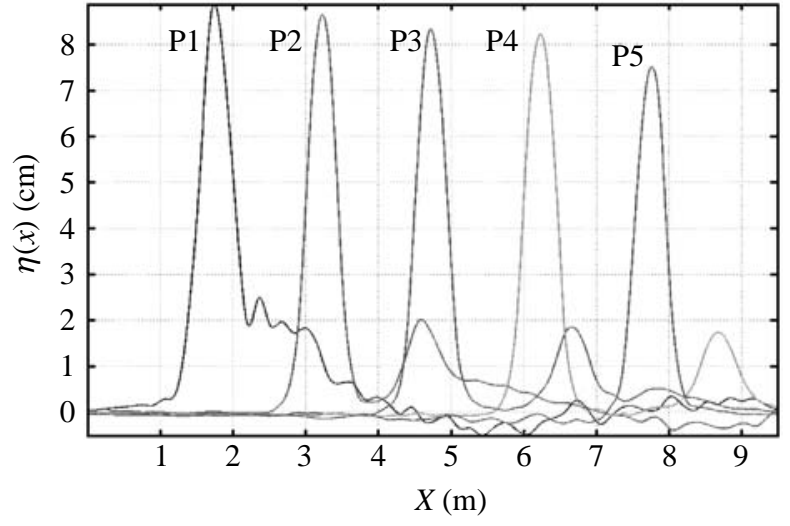

Fig. 9. Wave profiles versus distances along the flume, showing also the interval between leading the soliton feature and subsequent smaller waves.

nental zone. The stratified system in deep water has an upper layer with lighter warm water which is much thinner than the lower layer in heavier cold water. However, over the continental shelf, a two layer fluid system may gradually become transitional, thus producing solitary wave inversion instead of perfect single solitary waveform.

\section{Damping}

A number of dissipative mechanisms (e.g. viscosity and friction $e t c$.) may contribute to the dissipation and absorption of wave energy; yielding in progressive attenuation of wave amplitude in the course of propagation. Hence, the scenario of a permanent waveform could not be realized in the laboratory even it was once referred by Koop and Butler [11] and Michallet and Barthelemy $[12,13]$. In order to quantify the amount of dissipation, decaying rates these two papers are calculated and results are tabulated in Table 2 . These values include the effects of viscosity and friction in a stratified system in the flume. Higher dissipating rate was experienced in a water-petroleum system than that in the fresh-brine water experiments. For 


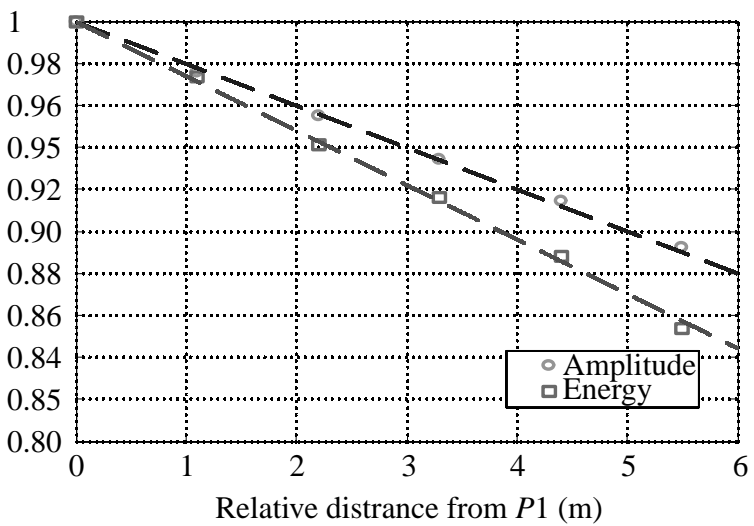

Fig. 10. Typical example of amplitude and energy attenuation of an ISW, showing normalized decaying rate with respect to the value at probe $P 1$ versus relation position. The symbols show values from 6 positions for a forward moving wave in a constant water depth of two-layer system.

instance, for the wave profiles (Figure 9) recorded by five ultrosonic probes equidistantly spaced along the flume, the damping rate in this run was in the same order as that reported by Ariyaratnam [1], perhaps due to the same fluid was used. The viscosity coefficient between brine and fresh water is respectively $v=1.17 \times 10^{-6}$ and $1.12 \times 10^{-6} \mathrm{~m}^{2} / \mathrm{s}$, respectively. In this test run, damping due to bottom friction only was considered, as viscosity effect might be weak in a fresh-brine water system. Previous laboratory experiments have showed that both the amplitude and energy of an ISW decay with distances in fresh-brine stratified fluid [1, 17]. In the present study, results indicate the slow decay rate with distance due to viscosity effect might be weak and hence could be reasonably ignored. As seen in Figure 10 , the amplitude and energy at position P1 to P5 normalized with respect to that at P1 versus from $\mathrm{P} 1$ distances suggest a linear decaying rate is appropriate. The rate of decay in wave amplitude was slightly smaller than that in energy.

\section{CONCLUSION}

Based on the thickness ratio $H_{1} / H_{2}$ in a stratified two-layer system, ISW form can be categorized as either elevation or depression type. Examples of the measured waveform for three different depth parameters $\left|H_{2}-H_{1}\right| / H$ indicate that a better soliton feature can be observed with larger value of depth parameters.

Dissipation in amplitude and energy occurs for internal solitary waves propagating in a wave flume. The amplitude of most internal solitary waves in the present experiments was found to decay approximate by $10 \%$ over a journey of 6 meters.

\section{ACKNOWLEDGEMENTS}

The authors are grateful for the research grants to JRCH from the National Science Council, Republic of China under Grant No. NSC 90-2611-M-110-014 and NSC 91-2611-M-110-004. They wish to thank Dr. H. Michallet, Laboratoire des Ecoulements Geophysiques et Industriels (LEGI), Institute National Polytechnique de Grenoble, France for his assistance in acquisition of the ultrasonic probes used in this study as well as MNDAS software system supplied by Tainan Hydraulics Laboratory, National Cheng Kung University, Taiwan.

\section{REFERENCES}

1. Ariyaratnam, J., "Investigation of Slope Stability under Internal Wave Action," Bachelor Thesis, Department of Environmental Engineering, University of Western Australia, Australia (1998).

2. Bogucki, D. and Redekopp, L.G., "A Mechanism for Sediment Resuspension by Internal Solitary Waves," Geophysical Research Letters, Vol. 26, pp. 1317-1320 (1999).

3. Bogucki, D. and Redekopp, L.G., "Resuspension and Boundary Mixing Stimulated by Long Internal Waves," Proceedings of 5th International Symposium on Stratified Flows, pp. 283-288 (2000).

4. Cacchione, D.A. and Southard, J.B., "Incipient Sediment Movement by Shoaling Internal Gravity Waves," Journal of Geophysical Research, Vol. 79, pp. 22372242 (1974).

5. Chen, C.Y., Hsu, J.R.C., Chen, H.H., Kuo, C.F., and Cheng, M.H., "The Evolution of a Generated Internal Solitary Wave by Gravity Collapse," Journal of Marine Science and Technology, Vol. 15, pp. 1-7 (2007).

6. Chen, C.Y., Hsu, J.R.C., Chen, H.H., Kuo, C.F., and Cheng, M.H., "Laboratory Observations on Internal Solitary Wave Evolution on Steep and inverse Uniform Slopes," Ocean Engineering, Vol. 34, pp. 157-170 (2007).

7. Chen, C.Y., Hsu, J.R.C., Cheng, M.H., Chen, H.H., and Kuo, C.F., "An Investigation on Internal Solitary Waves in a Two-Layer Fluid: Propagation and Reflection from Steep Slopes," Ocean Engineering, Vol. 34, pp. 171-184 (2007).

8. Chen, C.Y., Hsu, J.R.C., Kuo, C.F., Chen, H.H., and Cheng, M.H., "Laboratory Observations on Internal Wave Evolution Over a Submarine Ridge," China Ocean Engineering, Vol. 20, pp. 61-72 (2006).

9. Hegge, B.J., and Masselink, G., "Spectral Analysis of Geomophic Time Series: Auto-Spectrum," Earth Sur- 
face Processes and Landforms, Vol. 21, pp. 1021-1040 (1996).

10. Kao, T.W., Pan, F.S., and Renouard, D., "Internal Solitions on the Pycnocline: Generation, Propagation, Shoaling and Breaking over a Slope," Journal of Fluid Mechanics, Vol. 159, pp. 19-53 (1985).

11. Koop, C.G. and Butler, G., "An Investigation of Internal Solitary Waves in a Two-Fluid System," Journal of Fluid Mechanics, Vol. 112, pp. 225-251 (1981).

12. Michallet, H. and Barthelemy, E., "Ultrasonic Probes and Data Processing to Study Interfacial Solitary Waves," Experiments in Fluid, Vol. 22, pp. 380-386 (1997).

13. Michallet, H. and Barthelemy, E., "Experimental Study of Large Interfacial Solitary Waves," Journal of Fluid Mechanics, Vol. 366, No. 159-177 (1998).

14. Noble, M.A. and Xu, J.P., "Observations of Large-
Amplitude Sheared Cross-Shore Current Pulses over the Shelf Break, Santa Monica, CA," Marine Environmental Research, Special Issue on Santa Monica Bay, Vol. 56, pp. 127-149 (2003).

15. Segur, H. and Hammack, J.L., "Soliton Models of Long Internal Waves," Journal of Fluid Mechanics, Vol. 118, pp. 285-304 (1982).

16. Sherwin, T.J., Dale, A.C., Inall, M.E., and Jeans, D.R.G., "Linear and Non-Linear Internal Tides Around the European Atlantic Shelf Edge," Proceedings of 6th International Conference of off-Shore \& Polar Engineering, pp. 131-137 (1996).

17. Wessels, F. and Hutter, K., "Interaction of Internal Waves with a Topographic Sill in a Two-Layered Fluid," Journal of Physical Oceanography, Vol. 26, No. 1, pp. 5-20 (1996). 